\title{
Profissionais de saúde idosos: quais fatores fomentam e levam esse público a permanecer em atuação laboral após a aposentadoria?
}

Elderly health professionals: which factors foster and drive this public to remain in the workplace after retirement?

Profesionales de la salud mayores: ¿qué factores animan y llevan a este público a permanecer en el trabajo después de la jubilación?

Guilherme Mocelin

ORCID: https://orcid.org/0000-0001-9727-3619

Universidade de Santa Cruz do Sul, Brasil

E-mail: mocelinguilherme@gmail.com

Gabriele Zawacki Milagres

ORCID: https://orcid.org/0000-0003-3619-9317

Universidade de Santa Cruz do Sul, Brasil

E-mail: gabriele93.enf@gmail.com

Suzane Beatriz Frantz Krug

ORCID: https://orcid.org/0000-0002-2820-019X

Universidade de Santa Cruz do Sul, Brasil

E-mail: skrug@unisc.br

Hildegard Hedwig Pohl

ORCID: https://orcid.org/0000-0002-7545-4862

Universidade de Santa Cruz do Sul, Brasil

E-mail: hildegardpohl@outlook.com

Marcelo Carneiro

ORCID: https://orcid.org/0000-0003-3603-1987

Universidade de Santa Cruz do Sul, Brasil

E-mail:marceloc@unisc.br

\begin{abstract}
Resumo
Metodologia: estudo qualitativo exploratório descritivo realizado em um município do interior do estado do Rio Grande do Sul, com 16 profissionais de saúde de 60 anos de idade ou mais, tanto de atuação em Unidades Básicas de Saúde, quanto em hospitais. Para coleta de dados fez-se uso de entrevista semiestruturada e para análise dos dados, usou-se a Análise de Conteúdo. Resultados: destacaram-se quatro aspectos positivos no que se refere ao mantimento das atividades laborais no campo da saúde, sendo eles concernente a questões de satisfação profissional e pessoal, cultivo de relações sociais, mantimento das atividades de vida diária e no que diz respeito a contribuição com a sociedade. Sob outro prisma, observaram-se três pontos negativos que, de certa forma, lhes obriga a permanecer em atividade laboral, principalmente relacionadas às necessidades econômicas, receio de perdas identitárias e angústias quanto a invisibilidade e o isolamento social. Considerações Finais: assim sendo, trabalhar lhes traz ânimo e fomenta a qualidade de vida, todavia, emergem razões que lhes obrigam permanecer em ambiente laboral, para suprir necessidades humanas básicas e condições econômicas familiares.
\end{abstract}

Palavras-chave: Idoso; Aposentadoria; Qualidade de vida; Pessoal da saúde.

\begin{abstract}
Objectives: to analyze the discourses that promote the permanence of the elderly in the health labor field, after retirement. Methodology: descriptive exploratory qualitative study carried out in a city in the interior of the state of Rio Grande do Sul, with 16 health professionals aged 60 years or older, both working in Basic Health Units and in hospitals. For data collection, semi-structured interviews were used and for data analysis, Content Analysis was used. Results: four positive aspects were highlighted regarding the maintenance of work activities in the field of health, which concern issues of professional and personal satisfaction, cultivation of social relationships, maintenance of activities of daily living and with regard to contribution to society. From another perspective, there were three negative points that, in a way, oblige them to remain in work activity, mainly related to economic needs, fear of identity loss and anxieties about invisibility and social isolation. Final Considerations: therefore, working gives them encouragement and promotes quality of life, however, reasons emerge that oblige them to remain in a work environment, to meet basic human needs and family economic conditions.
\end{abstract}

Keywords: Aged; Retirement; Quality of life; Health personnel. 


\begin{abstract}
Resumen
Objetivo: analizar los discursos que incentivan la permanencia de las personas mayores en el ámbito laboral de la salud, después de la jubilación. Metodología: estudio cualitativo exploratorio descriptivo realizado en una ciudad del interior del estado de Rio Grande do Sul, con 16 profesionales de la salud de 60 años o más, tanto en Unidades Básicas de Salud como en hospitales. Para la recolección de datos se utilizaron entrevistas semiestructuradas y para el análisis de datos se utilizó Análisis de Contenido. Resultados: se destacaron cuatro aspectos positivos en cuanto al mantenimiento de las actividades laborales en el ámbito de la salud, que se refieren a cuestiones de satisfacción profesional y personal, cultivo de las relaciones sociales, mantenimiento de las actividades de la vida diaria y en cuanto a la contribución a la sociedad. Desde otra perspectiva, hubo tres puntos negativos que, de alguna manera, los obligan a permanecer en la actividad laboral, principalmente relacionados con las necesidades económicas, el miedo a la pérdida de identidad y las ansiedades por la invisibilidad y el aislamiento social. Consideraciones finales: por lo tanto, trabajar les da estímulo y promueve la calidad de vida, sin embargo, surgen razones que los obligan a permanecer en un ambiente laboral, para satisfacer las necesidades humanas básicas y las condiciones económicas familiares.
\end{abstract}

Palabras clave: Anciano; Jubilación; Calidad de vida; Personal sanitario.

\title{
1. Introdução
}

O envelhecimento é um fenômeno multidimensional em virtude das influências originadas através de aspectos socioculturais e do meio ambiente. No que concerne a saúde do idoso, a idade não é o único fator que deve ser levado em consideração, pois cada organismo responde a chegada dessa fase de um modo diferente. Ao contrário de algumas opiniões, é preciso desmantelar os estereótipos que restringem o processo da velhice com a perda da autonomia. Na maioria dos casos, a exposição aos fatores de risco são reflexos dos comportamentos inadequados que foram adquiridos ao longo do tempo. Esses, por sua vez, condicionam o indivíduo idoso a uma natureza vulnerável (Jardim; Medeiros; Brito, 2019). Em contrapartida, é possível conservar um estilo de vida saudável, viver bem e ter qualidade ao atingir esse estágio da vida. A combinação de bons hábitos, juntamente com estratégias de promoção de saúde e prevenção de doenças, são peças chaves para o bem-estar em qualquer ciclo da vida, especialmente para quem deseja uma vida longa (World Health Organization, 2017).

A atual conjuntura da dinâmica demográfica mundial projeta um crescimento acelerado no número de idosos (acima de 60 anos) no perfil populacional. Com a mudança na pirâmide etária surge a necessidade de (re)organizar o sistema e adaptar as políticas públicas existentes, para que seja possível acompanhar essa transição. Em termos, o envelhecimento populacional pode ser considerado um desafio, tanto para a saúde pública quanto para o mercado capitalista (Oliveira; Neto, 2018). Visto que, incontestavelmente, percebe-se um aumento na demanda dos serviços de saúde atrelado a idade avançada. Ainda nesse viés, em decorrência das transformações biológicas manifestadas pelo corpo, muitos idosos são obrigados a abandonar os espaços laborais, já que algumas funções exigem maior esforço físico. A diminuição nos percentuais produtivos pode impactar fortemente na economia, principalmente quando não há um planejamento prévio para lidar com esse panorama (Oliveira, 2019).

Alguns autores demonstram em seus estudos que existe uma parcela cada vez maior do contingente idoso, que decide continuar inserido no mercado de trabalho (Sá et al., 2011; Itaborai; Ricoldi, 2016). O crescimento na participação desse público no mundo laboral, ocorre por diversos motivos que vão desde a necessidade de aumentar a renda familiar ao prazer de se manter ativo e valorizado perante a sociedade. Ademais, o fato de envelhecer envolve uma mistura de sentimentos oriundos de uma cultura enraizada que denigre a imagem positiva do idoso, remetendo-o ao senso comum que compara esse momento da vida com a adversidade, o fracasso e até mesmo inutilidade. Esse pensamento, é fruto de um posicionamento preconceituoso sendo também conhecido como gerontofobia, que enxerga somente o lado negativo dessa etapa (Silva; Mendes, 2019). No entanto, há controvérsias que destoam da visão obscura acerca do tema e, que sobretudo, reconhecem a possibilidade em desfrutar da terceira idade de modo feliz, bem-sucedida e ativa (Freitas; Queiroz; Sousa, 2009; Freire, 2003). 
Uma questão primordial que abarca o processo de envelhecimento está diretamente vinculada à aposentadoria. Tratase de um marco que estabelece o afastamento dos idosos do campo de atuação. A dimensão do trabalho e a sua importância para o desenvolvimento da identidade pessoal, confronta a difícil decisão vivenciada por essa população. A permanência no espaço laboral geralmente está relacionada com algum significado importante para o idoso, seja o cultivo de bons relacionamentos interpessoais, satisfação profissional, ambiência acolhedora ou como forma de pertencimento social (Souza et al., 2020). Estudos comprovam que o engajamento desse público no desempenho de suas atividades torna-se essencial para a manutenção da estabilidade mental e psíquica (Ribeiro et al., 2018; Canizares; Filho, 2011). Contudo, a ideia da aposentadoria pode gerar diferentes percepções. Silva, Turra e Chariglione (2018), evidenciaram em sua pesquisa que existe certa preocupação e expectativa por parte dos aposentados em relação à nova realidade. Eles aproveitam para enfatizar a relevância de uma rede de apoio para o enfrentamento desse período, visando reduzir os índices de depressão ocasionados pela mudança na rotina.

De acordo com o contexto do trabalhador na área da saúde esse, pode ser visto como um modelo de integração entre os que o desenvolvem, considerado um trabalho vivo, ou seja, que depende dos diversos atores e níveis de conhecimentos compartilhados para a construção do produto final em ato, que resulta no cuidado humano (Merhy; Franco, 2008; Seixas et al., 2019). Esses idosos no campo laboral da saúde podem ser vistos como sujeitos de grande sabedoria em detrimento das largas experiências de vida e sabedoria que carregam. Entretanto, também podem ser observados à luz das fragilidades que emergem com a idade que avança (Marx, 1983). Desse modo, a dubiedade de sentimentos dessas atividades enigmáticas que circundam o campo da saúde e os constantes avanços tecnológicos, exigem tanto do sujeito idoso como dos que atuam em conjunto, constantes ponderamentos e ajustes, a fim de acompanhar a produção e às exigências do novo modelo de cuidado, prezando a qualidade assistencial (Sant'anna; Hennington, 2011). Assim sendo, buscar-se-á, analisar os discursos que fomentam a permanência do idoso no campo laboral da saúde, após a aposentadoria.

\section{Metodologia}

Com o intuito de captar todas as formas e expressões que se articulam com a vivência humana e o cenário, o presente estudo se debruça acerca da compreensão e aprofundamentos desses aspectos. Fatos que o conduzem pelo norte qualitativo exploratório descritivo, permitindo, de maneira holística a compreensão, análise, reflexão e crítica acerca da essência fenomenológica abordada (Minayo, 2014; Lacerda; Ribeiro; Costenaro, 2018).

Como local do estudo elegeram-se três hospitais e 32 unidades da rede de Atenção Básica de uma cidade situada na região centro-leste no estado do Rio Grande do Sul, sendo que destes, um hospital recusou-se a participar. Diante desses apontamentos denota-se que tal localidade é dotada de uma população aproximada de 118.374 pessoas, sendo que destas 15.559 (13,15\%) são idosos, ou seja, conforme o último censo realizado em 2010. Tais estimativas revelam que, para o ano de 2021 esses números podem chegar a valores que ultrapassam a casa dos 130 mil habitantes, distribuídos em um território de $733,409 \mathrm{Km}^{2}$. A economia da localidade supracitada, baseia-se na agricultura - especialmente no cultivo do tabaco - pecuária e setores industriais, diante dessa realidade destacam-se modelos de agricultura familiar até o abrigo de empresas multinacionais de grande impacto comercial (Instituto Brasileiro de Geografia e Estatística, 2010).

Quanto aos participantes da pesquisa, considerou-se profissionais de saúde com 60 anos de idade ou mais, tanto de atuação em Unidades Básicas de Saúde, quanto em hospitais. Ao levantar os dados acerca desses indivíduos foi constatado a existência de 25 sujeitos atuantes na Atenção Básica e 14 na atenção hospitalar, totalizando 39 profissionais idosos na área da saúde (Santa Cruz do Sul, 2021).

Como critérios de inclusão: ser trabalhador, profissional de saúde (das seguintes profissões - Enfermeiros, Médicos, 
Fisioterapeutas, Nutricionistas, Psicólogos, Técnicos e Auxiliares de Enfermagem, Biomédicos, Educadores Físicos, Farmacêuticos, Auxiliares de Saúde Bucal e Odontólogos), com idade igual ou superior a 60 anos, atuantes na rede de saúde do Sistema Único de Saúde (SUS) do local estudado, independentemente do tempo de atuação, além disso, possuir Ensino Superior ou Técnico Completo. Como critério de exclusão: encontrar-se em afastamento devido a férias, folgas ou motivos de saúde, no momento da coleta e produção dos dados. Considerando os fatores apontados, a amostra final contou com 16 participantes idosos trabalhadores no ramo da saúde, sendo oito atuantes na atenção básica e oito na atenção terciária. Ainda, sob esse contingente, dois não aceitaram fazer parte da pesquisa e 19 se encontravam em afastamento, férias ou folgas no momento da realização da coleta de dados. Tais afastamentos se caracterizaram por motivos de resguardo à saúde em detrimento a pandemia da Covid-19.

Para o mantimento do anonimato e garantia dos preceitos éticos aos participantes da pesquisa, os profissionais foram, por hora designados simplesmente pela letra $\mathrm{P}$, seguidos da numeração arábica, dada conforme a ordem que as coletas estavam correndo. Conforme o exemplo que segue: P01; P02; P03 e assim sucessivamente até o P16.

De modo a atender ao objetivo do estudo presente, para a coleta de dados fez-se uso de entrevista semiestruturada, constituída de um perfil sociodemográfico e ocupacional composto por 12 questionamentos e perguntas abertas relacionadas aos fatores que culminam na permanência do profissional idoso no campo laboral da saúde. As entrevistas foram gravadas em áudio e posteriormente transcritas para análise (Lacerda; Ribeiro; Costenaro, 2018). Os dados foram produzidos e coletados entre final de janeiro e início de março do ano de 2020, nos próprios locais de trabalho desses profissionais idosos, em ambiente reservado e silencioso, evitando a interferência de terceiros nas entrevistas e com isso a indução nas respostas. Para melhor comodidade dos participantes, foram realizados agendamentos prévios via contato telefônico, a fim de definir o melhor horário e dia para tal. Para prosseguir com as prerrogativas éticas em pesquisa o Termo de Consentimento Livre e Esclarecido (TCLE) foi aplicado e explicado detalhadamente à cada participante, sendo que as entrevistas tiveram duração média de 15 minutos.

Para analisar os materiais produzidos a partir das coletas de dados e das observações registradas nos diários de campo, faz-se uso da técnica de Análise de Conteúdo (Bardin, 2011). Tal abordagem é pautada em três pressupostos básicos que permite de forma didática e contínua compreender e interpretar os achados, sendo elas: pré-análise; exploração do material e tratamento dos resultados; inferência e interpretação.

Em conformidade aos preceitos da Resolução 466 de 12 de dezembro de 2012 do Conselhos Nacional de Saúde (Brasil, 2013), que trata sobre pesquisas com seres humanos, o estudo presente foi submetido e aprovado pelo Comitê de Ética em Pesquisa da Universidade de Santa Cruz do Sul (CEP-UNISC) em 09 de janeiro de 2020, sob o parecer consubstanciado de número 3.796.951.

\section{Resultados e Discussão}

\section{Perfil sócio-ocupacional}

No que tange ao perfil sociodemográfico desses trabalhadores, a despeito do gênero, 13 se autodeclararam mulheres. Referente às idades, elas oscilaram de 60 a 76 anos, e o predomínio permaneceu na faixa dos 60 a 65 anos, com dez participantes da pesquisa. Por sua vez, quando questionados acerca do estado civil: seis eram casados; quatro solteiros; três viúvos e três divorciados.

Quanto em relação ao perfil sócio ocupacional identificou-se que 11 eram Técnicos de Enfermagem, quatro Médicos e um Dentista e nessa tangente, ao tempo de formação, estas variaram de cinco a 45 anos, considerando que oito participantes possuíam idade superior a 41 anos desde a obtenção do título acadêmico. Por outro lado, quando questionados sobre o tempo 
de trabalho, desde o primeiro emprego até o atual, denota-se que esses variaram de 31 a 54 anos, sendo oito com tempo superior a 41 anos de vida laboral. No tocante ao emprego atual e tempo de atuação no mesmo, os valores variaram de cinco e 45 anos e destes: três participantes possuíam entre cinco a dez anos; dois de 16 a 20; quatro de 31 a 40 e sete acima de 41 anos de trabalho no mesmo local.

Ainda no que se refere ao perfil sócio ocupacional destes 16 participantes da pesquisa, atinente ao turno de trabalho, nove atuavam no período da manhã e tarde, três somente pela manhã, três somente à tarde e três nos turnos da manhã, tarde e noite. Por sua vez no quesito carga horária de trabalho semanal, nove faziam de 21 a 36 horas, três entre 37 e 40 horas, três mais de 41 horas e um 20 horas ou menos. Do grupo atual, 11 não possuíam mais de um vínculo empregatício, quatro possuíam dois e um possuía três locais de atuação. Alusivo à aposentadoria, todos afirmaram receber os valores previdenciários e o tempo médio que prevaleceu foi de nove anos.

\section{Aposentadoria: limites ou possibilidades para idosos profissionais da saúde?}

O envelhecimento é um segmento do ciclo vital do ser humano e envolve fatores considerados cruciais para a manutenção da qualidade de vida do indivíduo, como dispor de boa condição de saúde e de um trabalho digno. O fato de envelhecer acontece de forma natural e heterogênea. Diante disso, pode-se dizer que as mutações sofridas pelo corpo, possuem interação com o ambiente e por isso, vão aquém da idade (Morato; Ferreira, 2020). Assim, é possível encontrar pessoas idosas com plena capacidade física e mental, que não percebem limitações impostas pelo tempo e, no entanto, se sentem dispostas a continuar exercendo suas atividades normalmente.

Ainda que a temática referente à inserção e permanência do idoso no mercado de trabalho seja escassa, é inevitável reconhecer que o número de idosos que desejam seguir atuando nesse meio mesmo após a concessão da aposentadoria é expressivo em nosso país. A realidade demonstra que os valores previdenciários não estão sendo suficientes para suprir as necessidades básicas dessa população, que geralmente possuem custos com habitação, deslocamento, alimentação, substâncias farmacológicas e lazer (Ziger; Filippim; Beltrame, 2017). Face à importância em alargar as reflexões acerca do assunto e melhor entender quais são os sentimentos produzidos por essa população específica, fez-se o seguinte questionamento: qual o motivo de sua permanência no mercado de trabalho?

Caminhando ao encontro dos achados do presente estudo, evidenciou-se que os participantes destacaram quatro aspectos positivos no que se refere ao mantimento das atividades laborais no campo da saúde, sendo eles concernente a questões de satisfação profissional e pessoal, cultivo de relações sociais, mantimento das atividades de vida diária - mental e física - e no que diz respeito a contribuição com a sociedade. Sob outro prisma, observaram-se três pontos negativos que, de certa forma, lhes obriga a permanecer em atividade laboral, principalmente relacionadas às necessidades econômicas, receio de perdas identitárias e angústias no que tange a invisibilidade e o isolamento social.

Assim sendo, dez participantes acenaram para a importância do trabalho no cultivo das relações interpessoais, sentimentos valorativos e pertencimento a um grupo ativo do meio laboral, corroborando com os aspectos de vida ativa e os condicionantes promotores de saúde, considerando as falas que seguem:

Por que eu gosto, eu não sei fazer outra coisa, que ser só médico (P1).

[...] se eu for para de trabalhar agora é para ter uma "baita" depressão, eu acho (P3).

[...] então cheguei em um ponto na minha vida que eu achei agora eu posso fazer o que eu quero, porque um dia eu pensei, se a gente chegar num ponto da vida, olhar para trás e se arrepender do que fez, ou ter vontade de fazer e não fez, acho que ninguém vai ficar feliz, né! (P8). 
[...] eu não tenho doença nenhuma, me sinto forte, me sinto bem trabalhando, me sinto melhor trabalhando do que enfiada dentro de casa (P10).

O trabalho é compreendido como uma forma complexa de relações entre homens e cenário, que condiciona os sujeitos à ressignificação das realidades e compreensão das subjetividades que (des)constroem os fatores condicionantes do processo saúde-doença. Estar ajustado ao meio laboral de forma a proporcionar prazer aos indivíduos que os exercem, depende de uma vasta gama de fatores, que variam desde às condições do meio, até às relações pessoais entre os que atuam em conjunto. Como é passível de observar nas falas dos sujeitos, em comparativa com as ideias dos autores, esses sentimentos positivos emergem e podem oscilar de acordo ao vão momento que esses trabalhadores são atravessados, ou seja, não basta possuir o anseio por continuar em atividade laboral, se faz necessário o ponderamento de distintos fatores para o bem comum - empregado $\mathrm{x}$ empregador (Biancheto; Coltre; Mello, 2017).

Compreender as relações trabalhistas como formas que, além de resultar em produtos finais transformados, também edifica o sujeito que a desenvolve, exerce importante papel na autoestima e autorrealização dos trabalhadores idosos, devendo considerar os diferentes protagonistas diante dessas organizações de trabalho. A obtenção de realizações profissionais, como as que foram explicitadas pelos participantes da presente pesquisa pode resultar da compreensão que eles desenvolveram, acerca do significado de seus papéis para a sociedade, uma vez que, a sapiência desse contexto, costuma somar para obtenção desse sentimento de prazer relacionado com às experiências de vida laboral (Rocha et al., 2019).

Sob essa mesma esteira do pensamento, Marx (1983) afirma que a conjuntura entre dois meios/sujeitos é potência para modificação aos envoltos nesse processo. A interação entre o espaço e os atores, corrobora com a remodelação das realidades e perspectivas que os sujeitos sofrem e exercem enquanto atuam em meio laboral, ou seja, a dinâmica desenvolvida tangencia e influencia diretamente nas condições de vida e experiências que determinam os condicionantes de saúde-doença durante o curso da vida. Tais aspectos consideram o trabalho como sendo um importante aliado ao homem, sua convivência e participação na sociedade, levando em conta o contexto que evita a inércia do corpo e mente desses idosos. Sob essa mesma faceta, Dejours afirma e fortalece as reflexões que foram expostas, ponderando o trabalho como forma de dignificar os sujeitos e os relacionamentos (Dejours, 2007).

Mediante a tais explanações, podemos ainda acrescentar e correlacionar a importância em alinhar as metas que permeiam à Agenda 2030, visando através dos Objetivos de Desenvolvimento Sustentável (ODS) transformar as ações a nível global para melhorias dos aspectos fundamentais à vida, ou seja, que compõe os determinantes sociais da saúde. Tal proposta se articula com 193 países norteados pela Organização das Nações Unidas (ONU) os quais unidos, buscam atingir propósitos participativos comuns (Indicadores Brasileiros para os Objetivos de Desenvolvimento Sustentável, 2021). As interlocuções existentes entre os idosos em atividade no campo laboral da saúde e os ODS, procuram de modo efetivo entrelaçar e contribuir com as necessidades de todas as parcelas etárias emergentes em sociedade, tornando plausível as demandas que anseiam por mudanças e que possuem o intuito de proporcionar qualidade de vida e ampliar as condições holisticamente (Libório, 2021).

Dentre as metas e os objetivos elencados diante dessa proposta, são passíveis de relação com a presente temática os seguintes pressupostos, os quais buscam de forma conjunta e compartilhada a promoção de uma vida saudável de modo a estimular e proporcionar o bem-estar para todos os modelos de existência. A redução das desigualdades sociais e os protótipos equitativos de sociedade, tanto intra, quanto interpaíses, também pode ser direcionada aos fatores que regem as ações humanizadas e a busca por oportunidades uníssonas. Ainda sob esse caminho, quando atrelado às questões trabalhistas, visam de maneira positiva estimular o trabalho decente para todos(as) independentemente da idade e/ou fatores que se articulam com as formas laborais (Indicadores Brasileiros para os Objetivos de Desenvolvimento Sustentável, 2021). Assim sendo, a comunicação entre os ODS e o sujeito idoso, manifesta-se como forma de continuidade de direitos sociais e para melhorar o 
rumo das realidades.

Sob a outra face desse prisma, puderam ser notados nos discursos de seis participantes, menções relacionadas aos baixos valores previdenciários e necessidade de complemento de renda, além de medos pela perda da identidade social e o possível isolamento do convívio, como se configura nas falas a seguir:

\section{Baixos valores previdenciários}

[...] financeiro [...], eu também ajudo meus filhos, financeiramente [...] tenho netos (P6).

[...] a aposentadoria não é muito alta, então eu preciso trabalhar para ter mais uma renda. (P13)

[...] por causa do dinheiro, o salário sempre foi baixo, então sou obrigada a trabalhar, sou aposentada, ajudou, mas não chega. (P14)

[...] porque com a aposentadoria não sobrevive, tu vais sobreviver, mas de arrasto (P16).

\section{Anseios acerca do isolamento e invisibilidade social}

Se eu para de trabalhar eu não sei o que eu vou fazer (P1).

Eu não sou a diferente eu sou só mais velha (P8).

O que eu quero sozinha, os meus filhos não estão mais em casa [...] ficando só em casa a gente não tem o que fazer o dia a dia (P15).

Com base nas considerações analisadas percebe-se que o fator financeiro interfere na tomada de decisão para a postergação da aposentadoria. A remuneração recebida pelos aposentados não tem sido suficiente ou é menor do que o ganho anterior, dificultando o mantimento do padrão e qualidade de vida dessa população. O impacto dos valores previdenciários acaba influenciando o trabalhador a continuar atuando, mesmo contra seus desejos, para conseguir suprir suas necessidades básicas e com o intuito de complementar a renda salarial mensal. Essa situação contradiz o verdadeiro sentido que o trabalho proporciona na vida do indivíduo, no momento em que fragmenta o sentimento de prazer e realização profissional, pela privação de escolha do idoso, já que em alguns casos não deseja continuar trabalhando (Figueira et al., 2017).

Embora a aposentadoria seja considerada uma espécie de benefício, seus rendimentos nem sempre são satisfatórios, principalmente para quem preza pela qualidade de vida. Nessa perspectiva, a aposentadoria pode ser assimilada de forma negativa quando está relacionada com a queda do poder aquisitivo, trazendo consequências desfavoráveis à saúde do indivíduo. Frente a esse cenário, torna-se fundamental que haja maiores investimentos e formulação de estratégias efetivas que possam solucionar os desafios da Previdência Social, para alçar melhores condições e oferecer maior segurança nessa fase da vida (Souza et al., 2020).

A partir da compreensão das relações que são constantemente observadas e geradas pelos sujeitos e, partindo da compreensão mais aprofundada dessas relações, entende-se que sofrem e exercem constantes influências na construção coletiva e nas formas de ver e encarar essas realidades. Tais conexões entre os espaços sempre foram consideradas como alvo de estudos para permitir maior sapiência da complexidade dos conhecimentos que giram em torno do ser humano e seus determinantes (Santos; Guimarães, 2020). Sob essa esteira do pensamento, e de forma não cartesiana, justificam-se os medos e anseios que puderam ser vistos nas falas dos participantes, onde reforçam a importância para tais, nos mantimentos das relações e os impactos que essas causam para suas vidas.

Sentir-se membro de um espaço corrobora com aspectos de qualidade de vida e abarca os conceitos que se articulam com os modelos e condições, outrora descritos como potência geradoras de norte no curso da experiência humana, ou seja, as tomadas de decisões e os padrões de vida, doravante, determinam-se diretamente pelos fatores vivenciados enquanto relações. Ao passo que, abandonar esses laços, pode resultar em perdas de identidades e direcionar o homem ao adoecimento, uma vez 
que, consideram-se imprescindíveis as relações e subentende-se que a existência é rodeada destes encontros e nos padrões de vida em grupo (Oliveira; Santos, 2020).

Os sentimentos, desvalorização e despertencimento do idoso, atravessa a história humana, apresentando diversos sentidos de acordo com as tradições culturais de cada aglomerado populacional. Compreender a humanidade de forma isolada é convidativo para erros quando se analisam padrões em diferentes cenários, justamente pelos fatores que geram influências diante de todas elas e a construção que fora responsável por sua chegada a vão momento (Dutra; Coelho, 2020). Assim sendo, estar ajustado ao mercado laboral no campo da saúde e sentir-se parte deste, são conceitos que carecem de largas compreensões e não são sinônimos, encontrando-se à luz das percepções enquanto homens em sociedade e seus papéis para o indivíduo, família, instituições empregadoras, mercado do capital e conexões humanas/ambientais geradas e absorvidas. A valorização da pessoa idosa ainda necessita de importantes modelos que conduzam à compreensão de que o processo de envelhecimento caminha aceleradamente e carece absorver, manter ou abarcar essa população diante das necessidades, fragilidades e potencialidades a serem exploradas.

\section{Considerações Finais}

As percepções que cada sujeito formula em sua realidade se articula com uma gama de fatores que dependem diretamente das relações e encontros que são gerados de maneira ininterrupta, assim sendo, a aposentadoria, pode ser observada sob distintos prismas quando se apresenta à pessoa idosa trabalhadora da saúde. Trabalhar por fatores que lhe trazem ânimo a vida e fomenta a qualidade e aspectos de saúde são importantes pontos que puderam ser vistos nos discursos dos participantes do presente estudo e caminham ao encontro da real preconização de uma vida ativa para o envelhecimento saudável. Todavia, emergem razões que lhes obrigam a permanecer em ambiente laboral, de forma involuntária, para suprir, por vezes, necessidades humanas básicas e condições econômicas familiares.

Envelhecer, faz menção a um processo natural do curso da vida e, compreender esse processo a luz de fatores científicos e senescentes, requer amplos ponderamentos sociais a fim de promover e assimilar as mudanças que batem à porta do novo perfil etário, trazendo consigo, necessidades, fragilidades e potencialidades que hão de ser (re)vistas perante o novo cenário populacional e laboral. No entanto, estudos concernentes a essa temática, são imprescindíveis para o aprofundamento, continuidade e disseminação de conhecimentos acerca de outras localidades e contextos, haja vista, as lacunas existentes no que tange esses estudos, principalmente no que se refere aos trabalhadores idosos na área da saúde.

\section{Agradecimentos}

Agradecemos a Coordenação de Aperfeiçoamento de Pessoal de Nível Superior (CAPES) e o Conselho Nacional de Desenvolvimento Científico e Tecnológico (CNPq) pela concessão das bolsas de mestrado modalidade I e II e ao Programa de Pós-graduação - mestrado e doutorado - em Promoção a Saúde (PPGPS) da Universidade de Santa Cruz do Sul (UNISC).

\section{Referências}

Bardin, L (2011). Análise de Conteúdo. (4a ed.). Edições 70.

Biancheto, J. M.; Coltre, S. M.; \& Mello, G. R. (2017). Study on the valuabl factor of work. Rev capital científico, 15 (3), 1-13.

Brasil (2013). Resolução no 466, de 12 de dezembro de 2012. Dispõe sobre pesquisas em seres humanos e atualiza a resolução 196 [internet]. Conselho Nacional de Saúde. Brasília, https://conselho.saude.gov.br/resolucoes/2012/Reso466.pdf.

Canizares, J. C. L.; \& Filho, W. J. (2011). Fatores de risco à senilidade na transição à aposentadoria. Rev Bras Geriatr Gerontol, 14 (3), $425-32$.

Dejours, C (2007). A carga psíquica do trabalho. In: Betiol, MIS. Psicodinâmica do trabalho: contribuições da Escola Dejouriana à análise da relação prazer, sofrimento e trabalho. São Paulo: Atlas. 
Research, Society and Development, v. 10, n. 16, e11101623021, 2021

(CC BY 4.0) | ISSN 2525-3409 | DOI: http://dx.doi.org/10.33448/rsd-v10i16.23021

Dutra, R. Q.; \& Coelho, I. B. (2020). They think we are invisible”: gender, outsourcing, and popular legal education. Rev Direito e Práx, 11 (4), $2359-85$.

Figueira, D. A. M.; Haddad, M. C. L.; Gvozd, R.; \& Pissinati, P. S. C. (2017). Retirement decision-making influenced by family and work relationships. Rev Bras Geriatr Gerontol, 20 (2), 207-15.

Freire, S. A (2003). Envelhecimento bem-sucedido e bem-estar psicológico. In: Neri, AL; Freire, SA. E por falar em boa velhice. Campinas: Papirus, 21-31.

Freitas, M. C.; Queiroz, T. A.; Sousa, J. A. V. (2009). O significado da velhice e da experiência de envelhecer para os idosos. Rev Esc Enferm USP, 44 (2), $407-12$.

Indicadores Brasileiros para os Objetivos de Desenvolvimento Sustentável (2021) [internet]. Brasília, https://odsbrasil.gov.br/.

Instituto Brasileiro de Geografia e Estatística (2010). A população brasileira envelhece em ritmo acelerado [internet]. Brasília, http://www.ibge. gov.br/home/presidencia/noticia s/noticia_visualiza.php?id_noticia=1272.

Itaborai, N. R.; \& Ricoldi, A. M. (Org.) (2016). Até onde caminhou a revolução de gênero no Brasil? ABEP.

Jardim, V. C. F. S.; Medeiros, B. F.; \& Brito, A. M. (2019). A view on the aging process: elderly's perception of old age. Rev Bras Geriatr Gerontol, 9 (2), 2534.

Lacerda, M. R.; Ribeiro, R. P.; \& Costenaro, R. G. S. (Org.) (2018). Metodologias da pesquisa para a enfermagem e saúde: da teoria à prática. (2a ed.), Moriá Editora.

Libório, T. R (2021). A importância dos ODS - Objetivos de Desenvolvimento Sustentável, no desafio da educação para os direitos humanos. Rev Interdisciplinar de Direitos Humanos, 9 (1), 275-96.

Marx, K. (1983). O capital: crítica da economia política. Abril Cultural.

Merhy, E. E.; \& Franco, T. B. (2008). Trabalho em saúde. In: Pereira, IB; Lima, JCF (Org.). Dicionário da educação profissional em saúde. Rio de Janeiro: EPSJV, 247-432.

Minayo, M. C. S. (2014). O desafio do conhecimento: pesquisa qualitativa em saúde. (14a ed.), HUCITEC.

Morato, L. Z.; \& Ferreira, H. G. (2020). O mercado de trabalho para idosos: a consultoria como possibilidade de atuação. Rev Psicol Organ Trab, 20 (3), 1097-1104.

ODS. Indicadores Brasileiros para os Objetivos de Desenvolvimento Sustentável, 2021: https://odsbrasil.gov.br/.

Oliveira, A. A.; \& Neto, J. V (2018). A influência da previdência privada como renda complementar à aposentadoria e as contribuições para a economia brasileira: um estudo bibliográfico. REINPEC, 4 (2), 72-88.

Oliveira, A S (2019). Transição demográfica, transição epidemiológica e envelhecimento populacional no Brasil. Hygeia - Rev Bras Geografia Médica e da Saúde, 15 (32), 69-79.

Oliveira, M. S. S.; \& Santos, L. A. S. (2020). Dietary guidelines for Brazilian population: an analysis from the cultural and social dimensions of food. Ciênc Saúde Colet, 25 (7), 2519-28.

Ribeiro, P. C. C.; Almada, D. S. Q.; Souto, J. F.; \& Lourenço, R. A. (2018). Permanence in the labour market and life satisfaction in old age. Rev Ciên Saúde Colet, 23 (8), 2683-92.

Rocha, G. S. A.; et al (2019). Feelings of pleasure of nurses working in primary care. Rev Bras Enferm, 72 (4), 1036-43.

Sá, C. M. S.; Souza, N. V. D. O.; Caldas, C. P.; \& Lisboa, M. T. L. (2011). O idoso no mundo do trabalho: configurações atuais. Cogitare Enferm, 16 (3), 53642 .

Sant'anna, S. R.; \& Hennington, E. A. (2011). The micropolitics of living work in the act, ergology and popular education: a proposition of a device to train health workers. Rev Trab, Educ e Saúde, 9 (1), 223-244.

Santa Cruz do Sul (2021). Prefeitura municipal de Santa Cruz do Sul [internet]. Rio Grande do Sul, http://www.santacruz.rs.gov.br/links/busca.

Santos, D. G. G.; \& Guimarães, M. (2020). Pertencimento: um elo conectivo entre o ser humano, a sociedade e a natureza. REMEA, 37 (3), 208-23.

Seixas, C. T.; et al (2019). The power of the bond for Healthcare production: what guiding users teach us. Interface Comun Saude Educ, 23 (21), 1-14.

Silva, D. A.; \& Mendes, D. F. (2019). Da gerontofobia ao envelhecimento consciente e saudável. Psicol Saúde debate, 5 (2), 66-66.

Silva, M. M.; Turra, V.; \& Chariglione, I. P. F. S. (2018). Idoso, depressão e aposentadoria: Uma revisão sistemática da literatura. Rev Psic IMED, 10 (2), 11936.

Souza, L. B. C.; et al (2020). Elderly retirement postponement factors: an integrative literature review. Rev Ciên Saúde Colet, 25 (10), 3889-3900.

Souza, L. B. C.; et al (2020). Elderly retirement postponement factors: an integrative literature review. Ciênc Saúde Colet, 25 (10), 3889-900.

World Health Organization (2017). Global strategy and action plan on ageing and health [Internet]. Geneva, http://www.who.int/ageing/WHO-GSAP2017.pdf?ua=1.

Ziger, R; Filippim, E. S; \& Beltrame, V (2017). Perspectives of careers for elderly people in organizations. ReCaPe, 7 (3), 64-87. 